\title{
THE VACUUM ELECTROMAGNETIC FIELD AND THE ENERGETICS OF THE INTERGALACTIC MEDIUM
}

\author{
A. Rueda \\ Department of Physics \\ University of Puerto Rico at Humacao \\ P.O. Box 10058, College Station \\ Humacao, PR 00661
}

\begin{abstract}
The equilibrium distribution for a low-density infinite system of electromagnetically (EM) interacting particles placed in euclidean space in equilibrium with the Lorentz-invariant (LI) zero-point field (ZPF) is also LI with an energy density $\rho(E) d E \approx E^{-1} d E$. This result follows semiclassically as well as quantum theoretically (QT). Such an idea is exploited to propose a means for energizing the intergalactic medium (IGM) and thus explain the X-ray background (XRB) as thermal bremsstrahlung (TB) of a $3 \times 10^{8} \mathrm{~K}$ thermally excited electron gas confirming observation (see, e.g., Marshall et al. 1980; Boldt 1987).
\end{abstract}

\section{INTRODUCTION}

The proposal that the XRB is due to TB from a hot intergalactic plasma was analyzed by Field and Perrenod (1977) and by Taylor and Wright (1989). This proposal presents three difficulties, however (Field 1980): (i) energetics, (ii) clusters stability, and (iii) cosmological production of $D$ and He. This is avoided if instead of a hot IGM the XRB is due to unresolved point sources. It appeared plausible that much of the background below $3 \mathrm{keV}$ (Giacconi et al. 1979) was due to quasars. However, an XRB produced by discrete sources also presents severe difficulties (see, e.g., Field 1980; Fabian 1981; Young and Yu 1987). No class of sources in an uncontrived manner is able to give the spectrum. Clusters are too soft and galaxies too hard. Clusters are not bright enough by almost an order of magnitude, and active galaxies fail by a larger factor. Clusters do not meet the isotropy constraint on the density of unresolved prospective sources. Hence clusters have to be excluded and galaxies are required to perform peculiar strong early $(z \geq 0.5)$ evolution. Fabian (1981) estimated the discrete-unresolved-sources contribution in only $20 \%$ for the 3-10 keV range, and Young and Yu assigned only a $10 \%$ contribution to quasars around $2 \mathrm{keV}$.

\section{EQUILIBRIUM DISTRIBUTION IN THE ZPF}

Heating the IGM to $3 \times 10^{8} \mathrm{~K}$ requires considerable energy. Common proposals are: (i) accretion by macro-black holes in galactic centers, (ii) shock waves due to contraction of protoclusters, (iii) matter-antimatter annihilation, (iv) evaporation of primordial black holes, and (v) dark matter particle decay. We propose an alternative possibility: (vi) the EM fluctuations of the vacuum.

The ZPF has unique properties. Its spectral energy density,

$$
\rho(\omega) d \omega=\frac{\hbar \omega^{3}}{2 \pi^{2} c^{3}} d \omega,
$$

is homogeneous and isotropic in every inertial frame, i.e., equation (1) is LI. Furthermore, equation (1) is invariant under adiabatic compression (expansion) of a cavity and also under 
cosmic expansion (CE) (reviewed in Rueda 1989). Confined systems tend to resonate to the ZPF, e.g., a harmonically bound charged particle of characteristic frequency $\omega_{0}$ presents a delta-like equilibrium spectrum with the ZPF: $w(\omega) d \omega=0.5(\hbar \omega) \delta\left(\omega-\omega_{0}\right) d \omega$. The spectrum for an unconfined dilute system of infinitely many EM interacting particles in equilibrium with the ZPF in Euclidean space (in momentum $p$ representation) is $P(p) d p \approx E^{-1} d p$ which is a LI distribution (Boyer 1979), that for ultra-relativistic particles becomes $w(E) d E \approx E^{-1} \mathrm{e}^{\left(-E / \Omega T_{0}\right)} d E$, where $T_{0}=\mathrm{H}_{0}^{-1}$ is the Hubble time, and $\Omega$ is defined in equation (3). For mutually colliding particles, the differential flux is (Rueda 1989)

$$
J(E) d E \approx E^{-\zeta} \mathrm{e}^{\left(-E / \Omega T_{0}\right)} d E,
$$

with $\xi=1+\eta\left(\alpha^{2} c n / 2 \Omega m c^{2}\right)$, where $\eta$ is a numerical constant, $\alpha=\mathrm{e}^{2} / \hbar c$, and $\Omega$ can be calculated (Rueda 1984, 1986, 1989)

$$
\Omega=\frac{3}{5 \pi}\left(\Gamma \omega_{0}\right)^{2}\left[\frac{\hbar \omega_{0}}{m c^{2}}\right]\left(\hbar \omega_{0}\right) \omega_{0} .
$$

In the semiclassical case the result is exact, while the QT case of equation (3) is approximate with error smaller than $2 \%$ (Rueda 1989). $\Gamma=2 \mathrm{e}^{2} / 3 m c^{3}$ and $\omega_{0}$ depend on the particle model.

\section{X-RAY BACKGROUND BY A HOT IGM}

Under suitable conditions the above mechanism may energize the IGM. Protons and nuclei are accelerated and display particle density spectra with energy as in equation (2) that because $\Omega$ of equation (3) is fairly large, is seen as

$$
J(E) d E \approx E^{-\zeta} d E \text {. }
$$

If the particle density in the IGM is low, $n \cong 10^{-6} \mathrm{~cm}^{-3}, \xi$ should be close to $1, \xi \geqq 1$. Electrons are energized by the protons of the IGM and as the number densities of electrons and protons are small, $n_{\mathrm{e}} \cong n_{\mathrm{p}} \cong 10^{-6} \mathrm{~cm}^{-3}$, protons move ultra-relativistically and the energy dissipation mechanisms for electrons (CE, TB inverse Compton collisions with $2.7 \mathrm{~K}^{\circ}$ photons) are inefficient, electrons should tend to equilibrate among themselves around a temperature $T$ displaying a TB spectrum. This mechanism has been examined for the case of cosmic ray primaries acceleration (see, e.g., Rueda 1989).

\section{REFERENCES}

Boldt, E. 1987, Phys. Rep., 146, 216.

Boyer, T. H. 1979, Phys. Rev., 20, 1246.

Fabian, A. C. 1981, Ann. NY Acad. Science, 375, 235.

Field, G. B. 1980, in Some Strangeness in the Proportion, ed. H. Wolf (Reading, Mass.: Addison Wesley), p. 308.

Field, G. B. and Perrenod, S. C. 1977, Ap. J., 215, 717.

Giacconi, R. et al. 1979, Ap. J., 234, L1.

Marshall, F. E. et al. 1980, Ap. J., 235, 4.

Rueda, A. 1984, Phys. Rev. A, 30, 2221.

Rueda, A. 1986, Nuovo Cimento, 96B, 64.

Rueda, A. 1989, Space Science Reviews, submitted.

Taylor, G. B. and Wright, E. L. 1989, Ap. J., 339, 619.

Young, E. C. M. and Yu, K. N. 1987, Proceedings XX Int. Cosmic Ray Conf., Moscow, USSR-1987, eds. V. A. Kozyarivsky, et al. (Moscow: NAUKA), Vol. 1, pp. 181, 184, 188, and 199. 